La serie di Working Paper of Public Health (WP) dell'Azienda Ospedaliera di Alessandria è una serie di pubblicazioni online ed Open Access, progressiva e multi disciplinare in Public Health (ISSN: 2279-9761). Vi rientrano pertanto sia contributi di medicina ed epidemiologia, sia contributi di economia sanitaria e management, etica e diritto. Rientra nella politica aziendale tutto quello che può proteggere e migliorare la salute della comunità attraverso l'educazione e la promozione di stili di vita, così come la prevenzione di malattie ed infezioni, nonché il miglioramento dell'assistenza (sia medica sia infermieristica) e della cura del paziente. Si prefigge quindi l'obiettivo scientifico di migliorare lo stato di salute degli individui e/o pazienti, sia attraverso la prevenzione di quanto potrebbe condizionarla sia mediante l'assistenza medica e/o infermieristica finalizzata al ripristino della stessa.

Gli articoli pubblicati impegnano esclusivamente gli autori, le opinioni espresse non implicano alcuna responsabilità da parte dell'Azienda Ospedaliera "SS. Antonio e Biagio e Cesare Arrigo" di Alessandria.

La pubblicazione è presente in: Directory of Open Access Journals (DOAJ); Google Scholar; Academic Journals Database:

\section{Comitato Scientifico:}

Prof. Roberto Barbato

Dott.ssa Manuela Ceccarelli

Dott. Diego Gazzolo

Dott.ssa Federica Grosso

Prof. Marco Krengli

Prof.ssa Roberta Lombardi

Prof. Leonardo Marchese

Prof. Vito Rubino

Dott. Gioel Gabrio Secco

Dott. Paolo Tofanini
Dott. Giacomo Centini

Dott. Gianfranco Ghiazza

Dott.ssa Daniela Kozel

Dott. Marco Ladetto

Dott. Antonio Maconi

Dott. Alessio Pini Prato

Dott.ssa Mara Scagni

Dott.ssa Maria Elena Terlizzi

Dott.ssa Roberta Volpini il WP (i.e. peer review). L'utilizzo del peer review costringerà gli autori ad adeguarsi ai migliori standard di qualità della loro disciplina, cosi come ai requisiti specifici del WP. Con questo approccio, si sottopone il lavoro o le idee di un autore allo scrutinio di uno o più esperti del medesimo settore. Ognuno di questi esperti fornirà una propria valutazione, includendo anche suggerimenti per l'eventuale miglioramento, all'autore, così come una raccomandazione esplicita al Comitato editoriale su cosa fare del manoscritto (i.e. accepted o rejected).

Al fine di rispettare criteri di scientificità nel lavoro proposto, la revisione sarà anonima, così come l'articolo revisionato (i.e. double blinded).

\section{Diritto di critica:}

Eventuali osservazioni e suggerimenti a quanto pubblicato, dopo opportuna valutazione di attinenza, sarà trasmessa agli autori e pubblicata on line in apposita sezione ad essa dedicata.

Questa iniziativa assume importanza nel confronto scientifico poiché stimola la dialettica e arricchisce il dibattito su temi d'interesse. Ciascun professionista avrà il diritto di sostenere, con argomentazioni, la validità delle proprie osservazioni rispetto ai lavori pubblicati sui Working Paper of Public Health.

Nel dettaglio, le norme a cui gli autori devono attenersi sono le seguenti:

- I manoscritti devono essere inviati alla Segreteria esclusivamente in formato elettronico all'indirizzo e-mail dedicato

- A discrezione degli autori, gli articoli possono essere in lingua italiana o inglese. Nel caso in cui il manoscritto è in lingua italiana, è possibile accompagnare il testo con due riassunti: uno in inglese ed uno in italiano, cosi come il titolo;

- Ogni articolo deve indicare, le Keywords, nonché il tipo di articolo (i.e. Original Articles, Brief Reports oppure Research Reviews);

Comitato editoriale:

Dott. Antonio Maconi

Dott. Alfredo Muni

Dott.ssa Marinella Bertolotti

\section{Responsabile:}

Dott. Antonio Maconi

telefono: +39.0131.206818

email: amaconi@ospedale.al.it

\section{Segreteria:}

Mariateresa Dacquino, Marta Betti,

Mariasilvia Como, Laura Gatti

telefono: +39.0131.206192

email: mdacquino@ospedale.al.it; Igatti@ospedale.al.it

\section{Norme editoriali:}

Le pubblicazioni potranno essere sia in lingua italiana sia in lingua inglese, a discrezione dell'autore. Sarà garantita la sottomissione di manoscritti a tutti coloro che desiderano pubblicare un proprio lavoro scientifico nella serie di WP dell'Azienda Ospedaliera di Alessandria, purché rientrino nelle linee guida editoriali. II Comitato editoriale verificherà che gli articoli sottomessi rispondano ai criteri editoriali richiesti. Nel caso in cui lo si ritenga necessario, lo stesso Comitato editoriale valuterà l'opportunità o meno di una revisione a studiosi o ad altri esperti, che potrebbero o meno aver già espresso la loro disponibilità ad essere revisori per
- L'abstract è il riassunto dell'articolo proposto, pertanto dovrà indicare chiaramente: Obiettivi; Metodologia;

- Gli articoli dovrebbero rispettare i seguenti formati: Original Articles (4000 parole max., abstract 180 parole max., 40 references max.); Brief Reports (2000 parole max., abstract 120 parole max., 20 references max., 2 tabelle o figure) oppure Research Reviews (3500-5000 parole, fino a 60 references e 6 tabelle e figure);

- I testi vanno inviati in formato Word (Times New Roman, 12, interlinea 1.5). Le note, che vanno battute in apice, non possono contenere esclusivamente riferimenti bibliografici. Inoltre, la numerazione deve essere progressiva;

- I riferimenti bibliografici vanno inseriti nel testo riportando il cognome dell'Autore e l'anno di pubblicazione (e.g. Calabresi, 1969). Nel caso di più Autori, indicare nel testo il cognome del primo aggiungendo et al; tutti gli altri Autori verranno citati nei riferimenti bibliografici alla fine del testo.

- I riferimenti bibliografici vanno elencati alla fine del testo in ordine alfabetico (e cronologico per più opere dello stesso Autore).

Nel sottomettere un manoscritto alla segreteria di redazione, l'autore accetta tutte le norme qui indicate.
- Risultati; Conclusioni; 


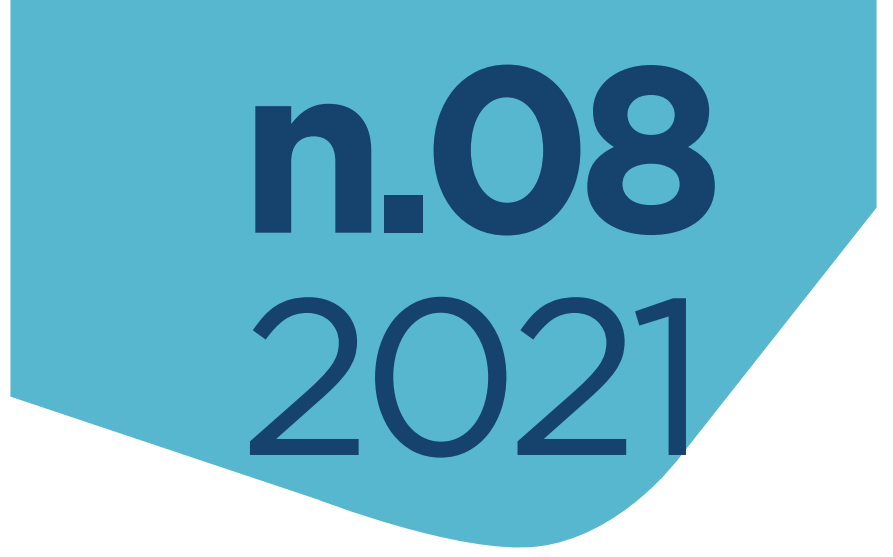

titolo

\title{
AUTOIMMUNITÀ E COVID-19: \\ UNO STUDIO PROSPETTICO MONOCENTRICO DI FOLLOW-UP
}

title

\author{
COVID-19 AND AUTOIMMUNITY: A SINGLE \\ CENTER PROSPECTIVE FOLLOW-UP STUDY
}

\section{autori \\ Maria Cristina Sacchi', Stefania Tamiazzo', Ernesto Cristiano Lauritano ${ }^{2}$, Ramona Bonometti ${ }^{3}$, Paolo Stobbione ${ }^{4}$, Antonio Maconi ${ }^{5}$.}

'SC Laboratorio Analisi, Settore di Autoimmunologia, AO SS Antonio e Biagio e Cesare Arrigo, Alessandria

2SS O.B.I. e Terapia Subintensiva, AO SS Antonio e Biagio e Cesare Arrigo, Alessandria

3 Medicina Interna, Ospedale Santo Spirito, Casale Monferrato, Alessandria ${ }^{4}$ SSD Reumatologia, AO SS Antonio e Biagio e Cesare Arrigo, Alessandria ${ }^{5} \mathrm{SC}$ Infrastruttura Ricerca Formazione Innovazione, AO SS Antonio e Biagio e Cesare Arrigo", Alessandria

tipologia

original article

keywords

COVID-19, malattie autoimmuni, SARS-Cov-2, prognosi, follow-up 


\section{ABSTRACT}

Obiettivi: In un nostro recente studio abbiamo riportato la presenza de novo di autoanticorpi in pazienti con diagnosi di COVID-19 ospedalizzati. Per verificare se questi autoanticorpi permangono nel tempo e/o portano allo sviluppo di malattie autoimmuni, abbiamo condotto uno studio di follow-up a 3 (†3) e 6 (†6) mesi dal ricovero.

Metodologia: Tredici dei 40 pazienti arruolati nel precedente studio hanno dato il consenso a partecipare a questo nuovo protocollo. Ai pazienti sono stati eseguiti gli stessi tests di autoimmunità effettuati al momento della diagnosi.

Risultati: In 9 pazienti continuano a persistere autoanticorpi circolanti a 6 mesi dalla diagnosi. Una paziente, negativa al momento del ricovero per tutti i tests di autoimmunità, ha sviluppato una forte positività per gli ANA e gli antigeni Mi2$\beta$ e Ku a †3 e a †6. Un altro paziente si è positivizzato a †3. Infine, due pazienti sono sempre rimasti negativi per tutto il periodo di monitoraggio.

Conclusioni: i nostri dati dimostrano che, dopo sei mesi, la presenza di autoanticorpi permane ancora nella maggior parte dei pazienti. Ulteriori indagini saranno necessarie al fine di poter verificare se questi pazienti si negativizzeranno nel tempo o potranno sviluppare una sintomatologia clinica compatibile con l'insorgenza di una malattia autoimmune cronica. Inoltre, sarà importante capire, se l'alterazione del sistema autoimmune può essere considerata come uno degli effetti del coronavirus (long Covid-19). 


\section{ABSTRACT}

Objectives: In our recent study we reported the presence of autoantibodies in COVID-19 positive hospitalized patients. To verify whether these autoantibodies persisted over time and led to the development of chronic autoimmune diseases, we conducted a follow-up study at 3 (t3) and 6 (†6) months after admission.

Methodology: Thirteen of 40 patients enrolled in the previous study gave their consent to participate in this new protocol. The same autoimmunity tests performed at the time of diagnosis were carried out in these patients.

Results: In 9 patients, autoantibodies were still present at follow-up. Another one, negative at admission, developed strong positivity for ANA and Mi2- $\beta$ and Ku antigens at +3 and at 16 . Only one showed the presence of de novo circulating autoantibodies at t3. Finally, we observed that two patients were always negative for the whole observation period.

Conclusions: Our data show that after 6 months, the autoantibodies are still present in the majority of our enrolled population. Further investigations will be necessary in order to verify whether these patients will become negative over time or may develop clinical symptoms coesistent with autoimmune disease. Finally, It will be important to understand whether an alteration of the autoimmune system can be considered effects of coronavirus (long COVID-19). 


\section{INTRODUZIONE}

La variante di beta-Coronavirus, denominata SARS-CoV-2 (Severe Acute Respiratory Syndrome-Corona Virus-2) è stata isolata per la prima volta a Wuhan, alla fine del 2019, ed è l'agente responsabile della malattia respiratoria acuta denominata COVID-19 (Corona Virus Disease-2019). La gravità delle patologie derivanti dalle infezioni da coronavirus è molto variabile, dal momento che questi virus sono responsabili sia di alcune comuni sindromi da raffreddamento che di sindromi respiratorie severe come la SARS (Severe Acute Respiratory Syndrome) e la MERS (Middle East Respiratory Syndrome) (Du Toit, 2020; Rothan et al, 2020; Hu et al, 2020). Nel caso dei pazienti COVID-19, la sintomatologia principale è quella tipica dell'influenza (febbre, tosse, stanchezza e dispnea) ma, in taluni casi, può evolvere in quadri clinici molto severi andando incontro alla sindrome da disfunzione multiorgano e portare anche alla morte (Mokhtari et al, 2020; Gupta et al, 2020.; Ciaccio et al, 2020). Si è osservato, inoltre, come l'evoluzione dell'infezione da SARS-COV-2 risulti avere caratteristiche simili alla sindrome da rilascio di citochine (Chen et al, 2020; Chen et al, 2021 ).

Questi dati sono stati confermati in un nostro recente studio, in cui abbiamo osservato come l'aumento del valore di marcatori infiammatori, in particolar modo di interleuchina 6 (IL-6) possa essere considerato un criterio diagnostico affidabile per identificare soggetti con una prognosi peggiore (Sacchi et al, $2021)$.

Sebbene l'eziopatogenesi delle malattie autoimmuni sia molto complessa e i meccanismi sottostanti debbano ancora essere completamente descritti, è noto come diversi agenti infettivi, batterici, virali o fungini possano rappresentare un trigger per lo sviluppo di malattie autoimmuni (Pesce et al, 2009).

Recenti lavori hanno descritto la presenza di diversi tipi di autoanticorpi in pazienti con COVID-19 (Zhang et al, 2020; Vlachoyiannopoulos et al, 2020; Pascolini et al, 2021; Liu et al, 2021). In aggiunta, diversi studi suggeriscono una possibile associazione tra l'infezione da SARS-CoV-2 e lo sviluppo di alcune patologie autoimmuni come il Lupus Eritematoso Sistemico (LES) (Bonometti et al, 2020), la sindrome di Guillain-Barrè (Soltani et al, 2019), la sindrome di Miller 
Fisher (Truong et al, 2020) la porpora trombocitopenica immune (Molinaro et al, 2020) e la malattia di Kawasaki (Ehrenfelda et al, 2020).

Tuttavia, ad oggi, i dati riportati sono ancora controversi; pertanto, al fine di poter comprendere se la presenza de novo di biomarcatori autoimmuni possa essere spiegata come un epifenomeno, una cross reazione, una patologia preesistente non diagnosticata o una nuova patologia autoimmune scatenata da COVID19, risulta fondamentale eseguire studi di follow-up.

Durante la prima ondata della pandemia da SARS-COV-2, abbiamo indagato l'assetto autoimmune di pazienti ricoverati con un quadro clinico severo, al fine di verificare una possibile correlazione tra presenza autoanticorpale e fenotipo clinico. Abbiamo così arruolato 40 pazienti tra i 20 e 97 anni $170 \%$ maschi e 30\% femmine) che erano stati ricoverati nel reparto di medicina sub-intensiva. Nessuno di questi pazienti presentava anamnesi di patologia autoimmune o sintomatologia che potesse esserne correlata prima del ricovero. Di questi pazienti sono stati studiati i più comuni autoanticorpi ed è stato evidenziato una correlazione tra infezione da SARS-CoV-2 e lo sviluppo di alcuni autoanticorpi. In particolare, quelli statisticamente significativi sono risultati essere: anticorpi antinucleo (ANA), anticorpi anti-citoplasma dei neutrofili (X-ANCA) e anticorpi anti Saccaromices cerevisiae (ASCA IgA). Pertanto, nel nostro studio abbiamo messo in evidenza che potrebbe esserci una correlazione tra la risposta a SARS-CoV-2, la presenza di autoanticorpi e l'outcome clinico (Sacchi et al, 2021). Al fine di verificare se la presenza autoanticorpale fosse transitoria, perdurasse nel tempo ed eventualmente fosse correlata allo sviluppo di patologie autoimmuni, abbiamo eseguito uno studio di follow-up su 13 dei 40 pazienti inizialmente arruolati. I pazienti sono stati testati a †3 e †6 dall'ospedalizzazione per i medesimi autoanticorpi considerati al t0. Questo lavoro ci ha permesso di osservare che la maggior parte dei pazienti ha mantenuto dopo 6 mesi un profilo autoanticorpale alterato. 
MATERIALI E METODI

\section{Approvazione Comitato Etico}

Questo studio osservazionale monocentrico prospettico è stato approvato dal Comitato Etico dell'Azienda Ospedaliera "SS Antonio e Biagio e Cesare Arrigo" di Alessandria. Questa ricerca è stata condotta in accordo con i principi etici della Dichiarazione di Helsinki.

\section{Disegno dello studio}

Sono stati arruolati per uno studio di follow-up 13 pazienti convalescenti COVID19 già analizzati al t0 (Sacchi et al., 2021). I time points considerati sono stati t3 e t6. I pazienti avevano un'età compresa tra 20 e 77 anni ed erano 6 uomini e 7 donne. Tutti i pazienti che hanno aderito allo studio hanno firmato il consenso informato.

\section{Test di autoimmunità}

Gli anticorpi anti-nucleo (ANA) e gli anticorpi anti-citoplasma dei neutrofili (ANCA) sono stati ricercati mediante l'immunofluorescence indiretta (IIF) usando i kit EUROIMMUN. Gli ANA sono stati individuati attraverso tre diluizioni seriali (1:80; 1:160; 1:320). I test di conferma sono stati eseguiti con la tecnologia line-blot usando i kit: EUROLINE Myositis DL-1530-4G e EUROLINE Scleroderma DL-1532 G. EUROLINE Myositis è specifico per i seguenti antigeni: Mi-2 alpha, Mi-2 beta, TIFlg, MDA5, NXP2, SAE1, KU, PM-SCl100, PM-SCl75, Jo-1, SRP, PL-7, PL-2, EJ, OJ, Ro-52. EUROLINE Scleroderma include questi antigeni: Scl-70, CENP A, CENP B, RP11, Rp155, fibrillarin, NOR90, Th/To, PM-Scl100, PM-Scl75, KU, PDGFR, Ro-52. Soltanto le bande con una intensità di segnale in EUROLineScan maggiore di 11 sono state considerate positive, secondo le istruzioni dell'azienda produttrice (EUROIMMUN, Luebeck, Germany). Gli ANCA sono stati determinati ad una diluizione di 1:20. Gli anticorpi anti-Saccharomyces cerevisiae (ASCA IgA e lgG), le proteine mieloperossidasi (MPO) e proteinasi 3 (PR3) sono stati analizzati mediante la metodica fluorescent enzyme immunoassay (FEIA) (Phadia 250- 
Thermoscientific, Freiburg, Germany). Gli antigeni nucleari estraibili (ENA) sono stati analizzati con la stessa teconologia FEIA, usando il pannello per le malattie connettivali CTD Screen (ENA screening): human recombinant UIRNP, SS-A/Ro, SS-B/La, centromero B, SCl-70, Jo-1, fibrillarina, RNA Pol III, Rib-P, PM-SCl, PCNA, Mi2 protein, Sm protein and DNA nativo purificato). Gli anticorpi antifosfolipidi (anticardiolipina, anti-ß2-glicoproteina lgA e lgG) sono stati analizzati usando il test di chemioluminescenza (ACL AcuStar, Instrumentation Laboratory, Bedford, USA). Valori superiori a 20 erano considerati positivi.

\section{RISULTATI}

In un nostro precedente studio retrospettivo osservazionale (Sacchi et al., 2021) abbiamo analizzato 40 pazienti sia dal punto di vista infiammatorio che immunologico, evidenziando le alterazioni dei più comuni markers di flogosi e una prevalenza significativa di autoanticorpi ANA, ANCA, ASCA IgA (Figura 1). Tredici pazienti, arruolati nel precedente studio con un'età compresa tra i 20 e i 77 anni (media $=55,69$ anni), sono stati analizzati a $\$ 3$ e 16 mesi per gli stessi autoanticorpi considerati a t0. Nessuno è stato affetto, negli ultimi 5 anni, da una delle seguenti malattie: infezione da epatite B, cancro, immunodeficienza, malattie cardiache ischemiche, ictus, demenza, malattia epatica cronica, infezione da HIV, TVP, EP, malattia cerebrovascolare. II tempo medio di ricovero in ospedale è stato di 22,54 giorni. Nove pazienti (P1, P2, P4, P6, P7, P8, P1 1, P12, P13) sono stati dimessi con un'importante insufficienza respiratoria da polmonite interstiziale bilaterale COVID-19 correlata. P11 e P13 presentavano una grave insufficienza respiratoria di tipo 1; P12 mostrava una trombocitopenia, molto probabilmente indotta da eparina; P5 presentava una porpora trombocitopenica immune (idiopatica); P10 era dimesso con la presenza di anomalie interstiziali (tabella supplementare 1). 
Iabella supplementare. Quadro clinico dei 13 pazienti osservati durante il follow-up

\begin{tabular}{|c|c|c|c|c|c|}
\hline & $\begin{array}{c}\text { Età } \\
\text { (anni) }\end{array}$ & Sesso & $\begin{array}{l}\text { Patologie concomitanti al } \\
\text { momento del ricovero }\end{array}$ & $\begin{array}{l}\text { Durata } \\
\text { degenza } \\
\text { (giorni) }\end{array}$ & Diagnosi alla dimissione \\
\hline P1 & 59 & $M$ & NO & 10 & Polmonite interstiziale COVID-19 correlato \\
\hline P2 & 64 & $\mathrm{~F}$ & NO & 22 & $\begin{array}{l}\text { Grave insufficienza respiratoria derivante } \\
\text { dalla polmonite interstiziale bilaterale } \\
\text { COVID-19 }\end{array}$ \\
\hline P3 & 72 & $\mathrm{~F}$ & $\begin{array}{c}\mathrm{BPCO} \text {, diabete, malattia } \\
\text { renale cronica, fibrillazione } \\
\text { atriale, obesità, gammopatia } \\
\text { monoclonale, scompenso } \\
\text { cardiaco }\end{array}$ & 26 & n.d. \\
\hline P4 & 53 & $\mathrm{~F}$ & $\begin{array}{l}\text { Asma, } \\
\text { emicrania, trombosi venosa }\end{array}$ & 5 & $\begin{array}{l}\text { Grave insufficienza respiratoria derivante } \\
\text { dalla polmonite interstiziale bilaterale } \\
\text { COVID-19 }\end{array}$ \\
\hline P5 & 20 & $\mathrm{~F}$ & NO & 5 & Porpora trombocitopenica immune \\
\hline P6 & 67 & $\mathrm{~F}$ & ipertensione & 15 & Polmonite interstiziale COVID-19 correlato \\
\hline P7 & 36 & M & ipertensione & 43 & $\begin{array}{l}\text { Grave insufficienza respiratoria derivante } \\
\text { dalla polmonite interstiziale bilaterale } \\
\text { COVID-19 }\end{array}$ \\
\hline P8 & 59 & $\mathrm{~F}$ & ipertensione & 17 & $\begin{array}{l}\text { Grave insufficienza respiratoria derivante } \\
\text { dalla polmonite interstiziale bilaterale } \\
\text { COVID-19 }\end{array}$ \\
\hline P9 & 48 & $M$ & NO & 42 & Polmonite interstiziale COVID-19 correlata \\
\hline P10 & 59 & $M$ & NO & 52 & Anomalie interstiziali \\
\hline P11 & 77 & $\mathrm{~F}$ & $\begin{array}{l}\text { ipertensione, precedente } \\
\text { tumore al seno, glaucoma }\end{array}$ & 16 & $\begin{array}{l}\text { Insufficienza respiratoria di tipo I derivata } \\
\text { dalla polmonite interstiziale bilaterale } \\
\text { COVID-19 }\end{array}$ \\
\hline P12 & 55 & M & $\begin{array}{l}\text { Colica renale, neuroma } \mathrm{C} 3- \\
\mathrm{C4} \text {; discopatia lombare }\end{array}$ & 22 & $\begin{array}{l}\text { Grave insufficienza respiratoria derivante } \\
\text { dalla polmonite interstiziale bilaterale } \\
\text { COVID-19 e trmbocitopenia }\end{array}$ \\
\hline P13 & 63 & M & asma & 18 & $\begin{array}{l}\text { Insufficienza respiratoria di tipo I derivata } \\
\text { dalla polmonite interstiziale bilaterale } \\
\text { COVID-19 }\end{array}$ \\
\hline
\end{tabular}

Da punto di vista dell'autoimmunità, 9 dei 13 pazienti (P4, P5, P6, P7, P8, P9, P10, P12, P13) presentavano una positività per gli anticorpi ANA a t0 (Figura 2-A). 
Figura 2 A. anticorpi anti-nucleo (ANA)

\begin{tabular}{|c|c|c|c|c|c|c|}
\hline ID & to & pattern & $\begin{array}{l}1 \\
t\end{array}$ & pattern & 16 & pattern \\
\hline P1 & - & & - & & - & \\
\hline $\mathbf{P 2}$ & - & & - & & - & \\
\hline P3 & $-*$ & $\begin{array}{l}\text { (granulare 1:320; } \\
\text { citoplasmatico 1:320)* }\end{array}$ & + & $\begin{array}{l}\text { granulare 1:160; } \\
\text { citoplasmatico 1:160; } \\
\text { centrioli 1:320 }\end{array}$ & + & $\begin{array}{l}\text { centrioli 1:160; } \\
\text { citoplasmatico } 80\end{array}$ \\
\hline P4 & + & granulare 1:160 & + & $\begin{array}{l}\text { omogeneo 1:80; } \\
\text { granulare 1:80 }\end{array}$ & + & $\begin{array}{l}\text { omogeneo 1:80; } \\
\text { granulare 1:80 }\end{array}$ \\
\hline P5 & + & centrioli 1:160 & + & centrioli $1: 80$ & + & centrioli 1:80 \\
\hline P6 & + & $\begin{array}{l}\text { mid body 1:80; fuso } \\
\text { mitotico 1:80 }\end{array}$ & + & mid body $1: 80$ & + & $\begin{array}{l}\text { mid body } 1: 80 \text {; fuso } \\
\text { mitotico 1:80 }\end{array}$ \\
\hline P7 & + & citoplasmatico 1:80 & - & & - & \\
\hline P8 & + & omogeneo $1: 320$ & + & omogeneo $1: 320$ & + & omogeneo $1: 320$ \\
\hline Pq & + & citoplasmatico 1:80 & - & & - & \\
\hline $\begin{array}{l}\text { P1 } \\
0\end{array}$ & + & citoplasmatico 1:80 & - & & - & \\
\hline $\begin{array}{c}\text { P1 } \\
1\end{array}$ & - & & + & omogeneo $1: 320$ & $\begin{array}{l}\text { n. } \\
\text { d. }\end{array}$ & \\
\hline $\begin{array}{l}\mathbf{P 1} \\
2\end{array}$ & + & $\begin{array}{l}\text { citoplasmatico 1:80; } \\
\text { granulare } 1: 160\end{array}$ & + & citoplasmatico $1: 80$ & - & \\
\hline $\begin{array}{c}\text { P1 } \\
3\end{array}$ & + & citoplasmatico 1:160 & + & citoplasmatico $1: 80$ & + & $\begin{array}{l}\text { nucleolare 1:160; } \\
\text { citoplasmatico 1:160 }\end{array}$ \\
\hline
\end{tabular}

In totale, 6 pazienti (P3, P4, P5, P6, P8 e P13) mantenevano la positività durante il follow-up, sia a $\$ 3$ che a $\nmid 6$. Un caso particolare è rappresentato dalla paziente P3, che risultava negativa a tutti i test autoimmuni a t0; dal momento che le sue condizioni cliniche non miglioravano e continuava a essere dipendente dall'ossigenazione artificiale, 16 giorni dopo il ricovero con un tampone molecolare nasofaringeo per SARS CoV-2 negativo, veniva rivalutato il suo profilo autoimmune: si riscontrava, così, una significativa positività per gli ANA, con un pattern fluoroscopico citolasmatico e granulare (1:320). La stessa paziente, in 
corrispondenza di †3, mostrava un quadro fluoroscopico misto: granulare (1:160), citoplasmatico (1:160), centrioli (1:320) mentre al t6 perdeva il pattern granulare. Inoltre, con la tecnica di secondo livello Immunoblot, eseguita sia a $\$ 3$ che a +6 , si confermava la presenza di autoanticorpi diretti contro gli antigeni Mi2- $\beta$ e Ku. P4 mostrava un quadro granulare (1:160) al to a cui si aggiungeva quello omogeneo (1:80) a †3 e †6. P5 ne mostrava invece uno centriolare (1:160) che è stato mantenuto al follow-up, mostrando sempre una positività per gli ENA (CTD screen). P6 era caratterizzata da un pattern misto mid-body e fuso mitotico che è stato mantenuto anche al follow-up. P8 aveva un pattern omogeneo (1:320). P13 mostrava a to un pattern citoplasmatico $(1: 160)$ che si è mantenuto a t3 $(1: 80)$ e $16(1: 160)$, time points in corrispondenza del quale compariva anche quello nucleolare (1:160).

Tre pazienti P7, P9, 10 con un quadro citoplasmatico a t0, si negativizzavano a $t 3$ e t6. P12 era positivo solo a to con un quadro citoplasmatico-granulare (1:80) e a +3 con uno citoplasmatico (1:80). Solo $P 11$ si è positivizzato a $† 3$ sviluppando un quadro omogeneo; purtroppo non è stato possibile studiarlo a †6. Infine, P1 e P2, negativi a t0, sono rimasti tali anche durante il follow-up.

Per quanto riguarda gli altri autoanticorpi analizzati (Figura 2-B): Pl ha conservato la positività per gli ENA per tutto il periodo di osservazione; P9 e P10 mostravano gli autoanticorpi X-ANCA a t0; P10 presentava anche gli ASCA IgG in tutti e tre i time points $(\uparrow 0, \uparrow 3, \uparrow 6)$, mentre gli ASCA IgA, borderline a $t_{0}$, si sono successivamente positivizzati a †3 e †6. P1 1 è risultato positivo per ASCA lgA e lgG solo a t0, mentre P12 ha sviluppato questi autoanticorpi durante il follow-up.

Nessuno dei 13 pazienti ha mostrato la presenza di: autoanticorpi anti fosfolipidi (anti-ß2glicoproteina, anti-cardiolipina), anti PR3 e anti MPO (dati non mostrati). 
Figura 2 B. Altri autoanticorpi

\begin{tabular}{|l|c|c|c|c|c|c|c|c|c|c|c|c|}
\hline \multirow{2}{*}{ ID } & \multicolumn{3}{|c|}{ ENA } & \multicolumn{3}{|c|}{ X-ANCA } & \multicolumn{3}{c|}{ ASCA IgA } & \multicolumn{3}{c|}{ ASCA IgG } \\
\cline { 2 - 14 } & t0 & †3 & †6 & t0 & †3 & †6 & t0 & †3 & †6 & t0 & †3 & †6 \\
\hline P1 & + & + & + & - & - & - & - & - & - & - & - & - \\
\hline P2 & - & - & - & - & - & - & - & - & - & - & - & - \\
\hline P3 & - & - & - & - & - & - & - & - & - & - & - & - \\
\hline P4 & - & - & - & - & - & - & - & - & - & - & - & - \\
\hline P5 & - & - & - & - & - & - & - & - & - & - & - & - \\
\hline P6 & - & - & - & - & - & - & - & - & - & - & - & - \\
\hline P7 & - & - & - & - & - & - & - & - & - & - & - & - \\
\hline P8 & - & - & - & - & - & - & - & - & - & - & - & - \\
\hline P9 & - & - & - & + & - & - & - & - & - & - & - & - \\
\hline P10 & - & - & - & + & - & - & b.I. & + & + & + & + & + \\
\hline P11 & - & - & n.d. & - & - & - & + & - & - & + & - & - \\
\hline P12 & - & - & - & - & - & - & - & + & + & - & + & + \\
\hline P13 & - & - & - & - & - & - & - & - & - & - & - & - \\
\hline
\end{tabular}

Figure 2. Ricerca di autoanticorpi durante il follow up in 13 pazienti affetti da SARS-CoV-2.

Tutti i 13 pazienti sono stati analizzati per i seguenti autoanticorpi: ANA, ENA, ANCA atipico (XANCA), ASCA IgA, ASCA IgG quando sono stati ricoverati (t0) e durante il follow up, dopo 3(†3) e 6 mesi(t6). La maggior parte dei pazienti presentava anticorpi anti nucleo al momento del ricovero $e$ in 5 di loro gli ANA persistevano anche durante il follow up (5/9). Una paziente (P3), negativo al t0, analizzato 16 giorni dopo il ricovero è risultata positiva per gli ANA. La tabella riassume i pattern trovati in ogni paziente nei diversi timepoint (A). Per quanto riguarda gli altri autoanticorpi, un paziente positivo per gli ENA al t0, è rimasto positivo durante il follow up. Due pazienti ( $P 9$ e 10) presentavano al to X-ANCA positivi e uno di loro (P10) era anche positivo per gli autoanticorpi ASCA IgG a tutti i tre time points, mentre gli ASCA IgA, borderline al to si sono positivizzati al $t 3$ e $t 6$. Un paziente ( $\mathrm{P} 11$ ) era positivo per gli ASCA IgA e IgG soltanto al t0, mentre un altro paziente (P12) ha sviluppato questi autoanticorpi durante il follow up.

(n.d. = non determinato; * = il paziente ha sviluppato gli autoanticorpi ANA 16 giorni dopo il ricovero; b.I.= borderline)

\section{DISCUSSIONE}

È noto come l'infezione da SARS-CoV-2 sia in grado di scatenare una forte e dannosa risposta immunitaria (Catanzaro et al, 2020); tuttavia il meccanismo patogenetico non è ancora stato completamente chiarito. Alcuni pazienti COVID-19 possono presentare sintomi debilitanti che perdurano per molti mesi dopo l'infezione iniziale (Long COVID-19) (Huang et al, 2021). Questa condizione è caratterizzata da stanchezza persistente, mal di testa, mancanza di respiro, anosmia, debolezza muscolare, febbre, nebbia cognitiva, tachicardia, disturbi intestinali e manifestazioni cutanee. In questa prospettiva, è fondamentale monitorare i pazienti COVID-19 dopo l'infezione virale per un lungo periodo così da comprendere il possibile risultato clinico. 
Recentemente, abbiamo riportato il caso di una donna di 85 anni che, dopo l'infezione da SARS-CoV-2, ha sviluppato trombocitopenia, effusione pleurica, proteinuria e basso complemento. La paziente presentava anche: una positività per gli ANA, caratterizzata da un quadro fluoroscopico misto (citoplasmatico 1: 160, omogeneo 1: 320 e granulare 1: 320), una positività per l'antigene Ku e per gli autoanticorpi X-ANCA. Quanto sopra elencato sono i criteri essenziali per diagnosticare il Lupus Eritematoso Sistemico (LES) (criteri EULAR 2019) (Aringer, 2019).

Questi dati sostengono fermamente l'idea che i pazienti COVID-19 positivi, con alterazioni dell'assetto autoimmune, debbano essere monitorati nel tempo al fine di osservare la possibile insorgenza di una malattia autoimmune cronica.

II nostro studio di follow-up ha evidenziato che solo 2 pazienti, P7 e P9, a 6 mesi dalla diagnosi si sono negativizzati, confermando il fatto che potesse trattarsi di una risposta correlabile all'infezione acuta. Di contro, un paziente che al to aveva un profilo autoimmune completamente negativo, rivalutato dopo 16 giorni, presentava anticorpi ANA e positività per gli antigeni M2- $\beta$ e Ku che permanevano anche a †3 e †6 (P3). Da un punto di vista clinico, questo paziente mostra ancora un'astenia muscolare persistente a livello del tronco, ma ulteriori indagini sono attualmente in corso per verificare la possibile insorgenza di una polimiosite (Sacchi et al, 2020).

Altri pazienti hanno mostrato una sintomatologia non correlata alla presenza di autoanticorpi. Per esempio, P8 ha mostrato un episodio transitorio di alopecia inquadrabile nel contesto di un telogen effluvium acuto post COVID-19 e non correlabile all'alopecia areata generalmente associata a condizioni autoimmuni (Forouzan et al, 2020). P4, P6, P13, nonostante abbiano mantenuto una positività al test ANA, non hanno mostrato sintomi compatibili con connettivopatia, in particolare con xerostomia xeroftalmia, ulcere al cavo orale, artriti, sinoviti o alterazioni cutanee. La giovane paziente P5, con positività agli ANA da pattern centriolare, ha sviluppato una iniziale piastrinopenia autoimmune che è stata trattata con steroidi ad alto dosaggio ed immunoglobuline (Molinaro et al, 2020). P12 ha sviluppato ipoacusia durante il 
follow-up. É noto come molte malattie autoimmuni possano provocare o essere associate a sordità autoimmune. La malattia dell'orecchio interno autoimmune (AIED) è stata definita come una condizione di perdita dell'udito neurosensoriale bilaterale, causata da una risposta "incontrollata" del sistema immunitario. AIED è considerata "primaria" quando l'orecchio interno è l'unico organo interessato; fino al 30\% dei casi, l'AIED è secondaria alla malattia autoimmune sistemica come il lupus eritematoso sistemico, l'artrite reumatoide, la sindrome di Sjögren o la granulomatosi di Wegener (Ciorba et al, 2018). Sarà quindi fondamentale monitorare il paziente a lungo termine al fine di determinare la natura dell'ipoacusia sviluppata.

Nei restanti soggetti, nonostante la persistenza di positività autoanticorpale, anche a titoli elevati, non è stata riscontrata nessuna correlazione clinica o sviluppo di patologie autoimmuni. In accordo con i dati di letteratura, infatti, la sola presenza di autoanticorpi non ha valore patogenetico (Kang et al, 2020). Tuttavia, risulta importante seguire nel tempo questi pazienti per capire se si negativizzeranno o se, a più lungo termine, potranno sviluppare una sintomatologia clinica.

In conclusione, il nostro studio è il primo volto ad indagare la persistenza di autoanticorpi nel tempo in pazienti COVID-19 e mette in evidenza come la maggior parte della popolazione arruolata mantenga un'allerazione dell'assetto autoanticorpale anche a distanza di 6 mesi dall'evento infettivo acuto. Sebbene il numero dei casi analizzati sia ridotto, i nostri risultati suggeriscono l'importanza di monitorare la presenza degli autoanticorpi a tempi più lunghi al fine di valutare il possibile sviluppo di patologie autoimmuni. 


\section{BIBLIOGRAFIA}

Aringer, M. "EULAR/ACR Classification Criteria for SLE." Seminars in Arthritis and Rheumatism 49, no. 3 (2019): S14-17. https://doi.org/10.1016/j.semarthrit.2019.09.009.

Bonometti R, Sacchi MC, Stobbione P, Lauritano EC, Tamiazzo S, Marchegiani A, Novara E, et al. "The First Case of Systemic Lupus Erythematosus (SLE) Triggered by COVID-19 Infection." European Review for Medical and Pharmacological Sciences 24, no. 18 (September 2020): 9695-97. https://doi.org/10.26355/eurrev_202009_23060.

Catanzaro M, Fagiani F, Racchi M, Corsini E, Govoni S, Lanni C. "Immune Response in COVID-19: Addressing a Pharmacological Challenge by Targeting Pathways Triggered by SARS-CoV-2." Signal Transduction and Targeted Therapy 5 , no. 1 (2020): 84. https://doi.org/10.1038/s41392-020-0191-1.

Guang C, Wu D, Guo W, Cao Y, Huang D, Wang H, Wang T, et al. "Clinical and Immunological Features of Severe and Moderate Coronavirus Disease 2019." Journal of Clinical Investigation 130, no. 5 (April 13, 2020): 2620-29. https://doi.org/10.1172/JCl137244.

Chen LYC, Quach TT. "COVID-19 Cytokine Storm Syndrome: A Threshold Concept." The Lancet Microbe 2, no. 2 (2021): e49-50. https://doi.org/10.1016/S26665247(20)30223-8.

Ciaccio M, Agnello L. "Biochemical Biomarkers Alterations in Coronavirus Disease 2019 (COVID-19)." Diagnosis 7, no. 4 (November 18, 2020): 365-72. https://doi.org/10.1515/dx-2020-0057.

Ciorba A, Corazzi V, Bianchini C, Aimoni C, Pelucchi S, Skarżyński PH, Hatzopoulos S. "Autoimmune Inner Ear Disease (AIED): A Diagnostic Challenge." International Journal of Immunopathology and Pharmacology 32 (2018): 205873841880868. https://doi.org/10.1177/2058738418808680.

Du Toit A. "Outbreak of a Novel Coronavirus." Nature Reviews Microbiology 18, no. 3 (2020): 123-123. https://doi.org/10.1038/s41579-020-0332-0.

Ebrahim Soltani Z, Rahmani F, Rezaei N. "Autoimmunity and Cytokines in GuillainBarré Syndrome Revisited: Review of Pathomechanisms with an Eye on 
Therapeutic Options." European Cytokine Network 30, no. 1 (March 1, 2019): 114. https://doi.org/10.1684/ecn.2019.0424.

Ehrenfeld M, Tincani A, Andreoli L, Cattalini M, Greenbaum A, Kanduc D, AlijotasReig J, et al. "Covid-19 and Autoimmunity." Autoimmunity Reviews 19, no. 8 (2020): 102597. https://doi.org/10.1016/j.autrev.2020.102597.

Forouzan P, Cohen PR. "Incipient Diabetes Mellitus and Nascent Thyroid Disease Presenting as Beard Alopecia Areata: Case Report and Treatment Review of Alopecia Areata of the Beard." Cureus, July 31, 2020. https://doi.org/10.7759/cureus.9500.

Gupta A, Madhavan MV, Sehgal K, Nair N, Mahajan S, Sehrawat TS, Bikdeli B, et al. "Extrapulmonary Manifestations of COVID-19." Nature Medicine 26, no. 7 (2020): 1017-32. https://doi.org/10.1038/s41591-020-0968-3.

Hu B, Guo H, Zhou P, Shi Z. "Characteristics of SARS-CoV-2 and COVID-19." Nature Reviews Microbiology 19, no. 3 (2021): 141-54. https://doi.org/10.1038/s41579020-00459-7.

Huang C, Huang L, Wang Y, Li X, Ren L, Gu X, Kang L, et al. "6-Month Consequences of COVID-19 in Patients Discharged from Hospital: A Cohort Study." The Lancet 397, no. 10270 (January 16, 2021): 220-32. https://doi.org/10.1016/501406736(20)32656-8.

Kang EH, Ha Y, Jong Lee Y. "Autoantibody Biomarkers in Rheumatic Diseases." International Journal of Molecular Sciences 21, no. 4 (February 18, 2020): 1382. https://doi.org/10.3390/ijms21041382.

Liu Y, Sawalha AH, Lu Q. "COVID-19 and Autoimmune Diseases." Current Opinion in Rheumatology 33, no. 2 (March 1, 2021): 155-62.

https://doi.org/10.1097/BOR.0000000000000776.

Mokhtari T, Hassani F, Ghaffari N, Ebrahimi B, Yarahmadi A, Hassanzadeh G. "COVID-19 and Multiorgan Failure: A Narrative Review on Potential Mechanisms." Journal of Molecular Histology 51, no. 6 (2020): 613-28. https://doi.org/10.1007/s10735-020-09915-3.

Molinaro E, Novara E, Bonometti R, Sacchi MC, Stobbione P, Lauritano EC, Boverio R. "Isolated Immune Thrombocytopenic Purpura in a Young Adult Covid-19 
Patient." European Review for Medical and Pharmacological Sciences 24, no. 20 (October 2020): 10850-52. https://doi.org/10.26355/eurrev 202010 23447.

Pascolini S, Vannini A, Deleonardi G, Ciordinik M, Sensoli A, Carletti I, Veronesi L, et al. "COVID-19 and Immunological Dysregulation: Can Autoantibodies Be Useful?" Clinical and Translational Science 14, no. 2 (2021): 502-8. https://doi.org/10.1111/cts.12908.

Pesce G. "Epidemiologia Delle Malattie Autoimmuni Organo-Specifiche." In II Laboratorio Nelle Malattie Autoimmuni d'organo, 14-36. Bologna: Esculapio, 2019.

Rothan HA, Byrareddy SN. "The Epidemiology and Pathogenesis of Coronavirus Disease (COVID-19) Outbreak." Journal of Autoimmunity 109 (2020): 102433. https://doi.org/10.1016/j.jaut.2020.102433.

Sacchi MC, Tamiazzo S, Stobbione P, Agatea L, De Gaspari P, Stecca A, Lauritano EC, et al. "SARS-CoV-2 Infection as a Trigger of Autoimmune Response." Clinical and Translational Science, January 21, 2021, cts.12953.

https://doi.org/10.1111/cts.12953.

Sacchi, MC, Tamiazzo S, Lauritano EC, Bonometti R. "Case Report of COVID-19 in an Elderly Patient: Could SARS-CoV2 Trigger Myositis?" European Review for Medical and Pharmacological Sciences 24, no. 22 (November 2020): 11960-63. https://doi.org/10.26355/eurrev 20201123857.

Truong J, Conley J, Ashurst J. "Miller-Fisher Syndrome: A Case Report and Review of the Literature." Clinical Practice and Cases in Emergency Medicine 4, no. 4 (November 2020): 653-55. https://doi.org/10.5811/cpcem.2020.7.48507.

Vlachoyiannopoulos PG, Magira E, Alexopoulos H, Jahaj E, Theophilopoulou K, Kotanidou A, Tzioufas AG. "Autoantibodies Related to Systemic Autoimmune Rheumatic Diseases in Severely III Patients with COVID-19." Annals of the Rheumatic Diseases 79, no. 12 (2020): 1661-63. https://doi.org/10.1136/annrheumdis2020-218009.

Zhang Y, Xiao M, Zhang S, Xia P, Cao W, Jiang W, Chen H, et al. "Coagulopathy and Antiphospholipid Antibodies in Patients with Covid-19." New England Journal of Medicine 382, no. 17 (April 23, 2020): e38.

https://doi.org/10.1056/NEJMc2007575. 
pubblicazione revisionata e approvata maggio 2021

disponibile online www.ospedale.al.it/ working-papers-wp 